\section{Acute right heart failure complicated by hypovolaemia}

The dangers of inducing diuresis in patients with pulmonary oedema and normal pulmonary wedge pressures have recently been described. We report a complementary case of acute right heart failure due to right ventricular infarction complicated by hypovolaemia. In this, right ventricular output may provide an insufficient preload for the left ventricle, and there is the apparent paradox of an overloaded right ventricle and an inadequately primed "hypovolaemic" left ventricle. Failure to recognise this may lead to inappropriate diuretic or inotropic treatment and potentially irreversible hypovolaemic shock.

\section{Case report}

A 66-year-old tailor presented with inferior myocardial infarction. He was normotensive and well perfused, with raised jugular venous pressure. Auscultation of the heart was normal and he had no signs of left ventricular failure. Because of the raised jugular venous pressure he underwent diuresis. Over three days he became hypotensive and oliguric. Jugular venous pressure remained raised in the absence of pulmonary congestion, and he developed ankle oedema. Inotropic drugs and diuretics produced no improvement.

A Swan-Ganz catheter was inserted (figure). Right atrial pressure was
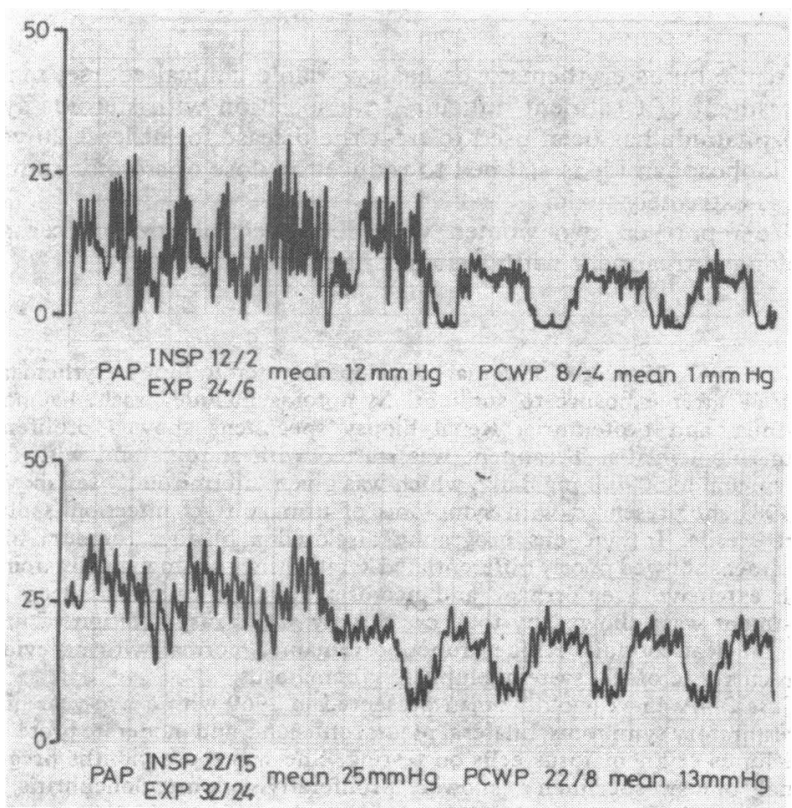

Left ventricular hypovolaemia due to right ventricular infarction. Top: Pressures on insertion of catheter. Bottom: Pressures after $250 \mathrm{ml}$ colloid given immediately.

$\mathrm{PAP}=$ Pulmonary artery pressure. $\mathrm{PCWP}=\mathrm{Pulmonary}$ capillary wedge pressure. Insp = Inspiration. Exp = Expiration.

raised with a mean systolic pressure of $12 \mathrm{~mm} \mathrm{Hg}$ from the mid-axilla (normal range 12-20) and a mean pulmonary capillary wedge pressure of $1 \mathrm{~mm} \mathrm{Hg}$ (normal range 8-12). These findings indicated isolated right ventricular failure with inadequate left ventricular filling. Fluid replacement restored the pulmonary wedge pressure, and his blood pressure and urine flow returned to normal. He recovered fully.

\section{Comment}

Clinical assessment of left ventricular filling is difficult. Radiologically the lung fields are clear when pulmonary capillary wedge pressure is below $18 \mathrm{~mm} \mathrm{Hg.}{ }^{2}$ Normal left ventricular filling pressure (reflected by pulmonary capillary wedge pressure) is $8-12 \mathrm{~mm} \mathbf{~ H g}$. When left ventricular function is mildly impaired, and particularly in biventricular failure, clear lung fields and a raised jugular venous pressure may co-exist. As the curves of ventricular function are flat the risk of pulmonary oedema rises with uncontrolled administration of fluids. Similarly, diuresis reduces ventricular filling with little fall in performance. Should left ventricular function be normal, however, the Starling curve lies to the left, filling pressures are low, and further reduction in pulmonary capillary wedge pressure appreciably reduces stroke volume.

The concept of the heart as two separate pumps is established, ${ }^{3}$ but clinical teaching still equates raised jugular venous pressure with biventricular failure; pulmonary embolus, pericardial tamponade, and cor pulmonale are mentioned in parenthesis, and right ventricular infarction has not yet reached the general textbooks. ${ }^{5}$ Consequently the unwary tend to attribute falling cardiac output in the presence of raised jugular venous pressure to myocardial failure, and the patient receives inotropic drugs and diuretics. The need for adequate left ventricular filling is often forgotten in the anxiety to avoid pulmonary oedema. A competent but hypovolaemic ventricle is therefore deprived of fluid and driven with inotropic drugs. Cardiac and urine outputs remain low. The patient undergoes diuresis and is rendered more hypovolaemic. Death is inevitable.

We propose that patients with right ventricular failure, poor perfusion, and normal chest $x$-ray films may be hypovolaemic. A $200 \mathrm{ml}$ bolus of colloid will improve hypovolaemia dramatically. This should be repeated until the patient is stable. Patients with biventricular failure will not respond. Deterioration in their condition may be reversed by diuresis or venesection. The Swan-Ganz catheter has made measurement of left-sided filling pressures accessible to most acute services, when fluid replacement may be tailored to provide a pulmonary capillary wedge pressure of $12-15 \mathrm{~mm} \mathrm{Hg}$.

We think that the life-saving potential of a fluid load in unsuspected hypovolaemia with right ventricular failure justifies fluid challenge when the diagnosis is in doubt.

1 Timmis A.D, Fowler MB, Burwood RJ, Gishen P, Vincent R, Chamberlain DA. Pulmonary oedema without critical increase in left atrial pressure in acute myocardial infarction. $\mathrm{Br}$ Med 7 1981;283:636-8.

${ }^{2}$ McHugh TJ, Forrester JS, Adler L, Zion D, Swan HJC. Pulmonary vascular congestion in acute myocardial infarction, haemodynamic and radiological correlations. Ann Intern Med $1972 ; 76: 29-33$.

${ }^{3}$ Bradley R. Studies in acute heart failure. London: Edward Arnold, 1976:1-7.

4 Sarnoff SJ, Berglund E. Starling's law of the heart studied by means of simultaneous right and left ventricular function curves in the dog. Circulation 1954;9:706-18.

s Rackley CE, Russell RO, Mantle JA, Rogers WJ, Papapietro SE, Schwarz KM. Right ventricular infarction and function. $A m \mathcal{F}$ Cardiol $1981 ; 48: 215-8$.

(Accepted 8 fanuary 1982)

Central Middlesex Hospital, London NW10 7NS

R J D GEORGE, MA, MRCP, registrar

Liver Unit, King's College Hospital, London SE5 9RS

D BIHARI, MA, MRCP, registrar

\section{Effect of cimetidine on portal hypertension in cirrhotic patients}

Cimetidine is claimed to reduce hepatic blood flow in normal subjects, but its effect on portal pressure has not been reported. If cimetidine also reduces hepatic blood flow in patients with cirrhosis then it would be an alternative to propranolol, which reduces both hepatic blood flow and portal pressure and protects against recurrent variceal bleeding in these patients. ${ }^{2}$ Cimetidine might have fewer side effects than propranolol, and long-term clinical trials of cimetidine prophylaxis for recurrent variceal haemorrhage in patients with cirrhosis have been advocated. We therefore studied the effect of intravenous cimetidine on the gradient between the wedged and free hepatic venous pressures, which is a reliable index of portal pressure, in patients with cirrhosis ${ }^{3}$ who had recently bled from oesophageal varices. 